\title{
Cascaded Perforates as One-Dimensional, Bulk Absorbers
}

\author{
T.L.Parrott* and M.G.Jones ${ }^{\dagger}$ \\ NASA Langley Research Center, Hampton, VA 23681-2199, USA
}

\begin{abstract}
$\mathbf{P}$ OROUS cell honeycomb liners for aircraft engine nacelles offer the possibility of exploiting extended reaction effects to improve liner attenuation bandwidth as generally attributed to the performance of bulk absorbers. This paper describes an analytical procedure, starting with an impedance prediction model for a single perforated plate, to estimate the bulk-absorber parameters for a cascade of such perforates - a first step to modeling a porous wall honeycomb structure. The objective is to build confidence in a lumped element impedance model, when applied to a uniformly-spaced set of porous plates to predict its "bulk" absorber properties. The model is based upon a modified version of the two-parameter flow resistance model of the form $A+B V_{\text {inc }}$, where $A$ and $B$ are physics-based, semi-empirical parameters that are adjusted to provide an optimum fit to a composite dataset from three plate porosities of $2.5,5$ and $10 \%$. The composite dataset is achieved by reformulating the two-parameter flow resistance model into a "reduced pressure drop coefficient" dependency on perforate hole Reynolds number. The resulting impedance model is employed to calculate surface impedance spectra for $N$ and $2 N$-layer perforate cascades. The well-known two-thickness method for experimental determination of bulk-absorber parameters is then applied to these "synthesized" data sets to predict the characteristic impedance and propagation constant for the perforate cascades. These results are then compared with experimental results reported in a companion paper.
\end{abstract}

\section{Nomenclature}

$\begin{array}{ll}A & \text { empirical constant } \\ A & \text { semi-empirical parameter group, }(a \mu t) /\left(2 \rho c \sigma C_{D} d^{2}\right) \\ B & \text { empirical constant, s/m } \\ B & \text { semi-empirical parameter group, }\left(k_{i}+k_{e}\right) /\left(2 c\left(\sigma C_{D}\right)^{2}\right), \mathrm{s} / \mathrm{m} \\ a & \text { laminar flow friction factor } \\ a_{\mathrm{opt}} & \text { optimized laminar flow constant } \\ c & \text { sound speed, } \mathrm{m} / \mathrm{s} \\ C_{D} & \text { hole discharge coefficient } \\ C_{D, \mathrm{opt}} & \text { optimized hole discharge coefficient } \\ d & \text { hole diameter, m } \\ C_{p d} & \text { aerodynamic pressure drop coefficient, } \Delta p /\left(\rho V_{\infty}^{2}\right) \\ f & \text { frequency, Hz } \\ k_{i} & \text { entrance loss coefficient } \\ k_{e} & \text { exit loss coefficient } \\ k & \text { wavenumber, m/s } \\ k_{x}, k_{y} & \text { wavevector compoents in x- and y-directions, m/s } \\ L_{\mathrm{cav}} & \text { cavity length, m } \\ p & \text { pressure, Pa } \\ p_{\mathrm{rms}} & \text { RMS incident acoustic particle velocity, m/s } \\ P r & \text { Prandtl number } \\ & \end{array}$

\footnotetext{
${ }^{*}$ Senior Research Scientist, Research \& Technology Directorate, Structural Acoustics Branch, Mail Stop 463; Tony.L.Parrott@NASA.GOV.

${ }^{\dagger}$ Senior Research Scientist, Research \& Technology Directorate, Structural Acoustics Branch, Mail Stop 463; Michael.G.Jones@ NASA.GOV, Senior member of AIAA.
} 


$\begin{array}{ll}R e_{h} & \text { hole Reynolds number, }(d / \mathrm{v}) V_{h} \\ R_{f} & \text { flow resistance, }\left(\Delta p / V_{\text {inc }}\right), \mathrm{kg} /\left(\mathrm{m}^{2} \mathrm{~s}\right) \\ t & \text { perforate thickness }(\text { hole length), } \mathrm{m} \\ V_{h} & \text { hole velocity, } V_{\mathrm{inc}} / \sigma, \mathrm{m} / \mathrm{s} \\ V_{\text {inc }} & \text { incident velocity, } \mathrm{m} / \mathrm{s} \\ V_{\text {rms }} & \text { RMS acoustic particle velocity, } \mathrm{m} / \mathrm{s} \\ V_{\infty} & \text { free-stream velocity, } \mathrm{m} / \mathrm{s} \\ \varepsilon & \text { classical end correction factor } 8 d /(3 \pi) \\ \sigma & \text { perforate open area ratio }(\text { porosity) } \\ \mu & \text { dynamic viscosity of air, } \mathrm{kg} /(\mathrm{ms}) \\ \nu & \text { kinematic viscosity of air, } \mathrm{m}^{2} / \mathrm{s} \\ \Delta p & \text { pressure drop across perforate, } \mathrm{kg} /\left(\mathrm{ms} \mathrm{s}^{2}\right) \\ \rho & \text { air density, kg/m } 3 \\ \theta & \text { normalized, specific acoustic resistance } \\ \chi & \text { normalized, specific acoustic reactance } \\ \chi_{m} & \text { normalized mass reactance } \\ \omega & \text { angular frequency, } 2 \pi f, \text { rad } / \mathrm{s} \\ \zeta & \text { normalized, specific acoustic impedance } \\ \zeta_{e} & \text { normalized, estimated, specific acoustic impedance } \\ \zeta_{\text {ter }} & \text { normalized, back plate (termination) impedance }\end{array}$

Abbreviations:

$\begin{array}{ll}\text { dB } & \text { decibels } \\ \text { EVRR } & \text { Extended Velocity Range Raylometer } \\ \text { NIT } & \text { Normal Incidence Impedance Tube } \\ \text { NLF } & \text { Nonlinear Factor } \\ \text { RMS } & \text { root-mean-square }\end{array}$

Note: The $e^{i \omega t}$ sign convention is used throughout this paper.

\section{Introduction}

DVANCES in materials technology continue to provide new possibilities for improved acoustic liner designs suit-
able to reduce noise emissions from aircraft engine nacelles. Historically, the simplest acoustic liners for this
application have been single-layer, perforate-over-honeycomb core structures. Such structures are locally-reacting,
and consequently are prone to exhibit strongly resonant behavior that limits their attenuation bandwidth. This band-
width limitation has been alleviated somewhat by incorporating multiple layers, which adds weight and mechanical
complexity. It is well known that bulk absorbing materials, such as foam and fiberglass, have relatively high absorp-
tion bandwidths. This can be attributed to their distributed dissipation and extended reaction, which tends to subdue
depth-related resonances. However, for mechanical and maintenance reasons, bulk absorbers have not been acceptable
for aircraft engine nacelle applications.
With recent advances in fabrication technologies, it is now possible to implement specified wall porosity into
honeycomb cell walls. This fabrication technology allows conventional perforate-over-honeycomb designs to take on
specified extended reaction behavior without complicating the basic mechanical design. Thus, the liner designer can
implement design trade-offs between the conventional locally-reacting, resonant absorbers and the extended-reacting
behavior associated with bulk absorbers. The physics underlying these differences is illustrated in the sketches of
figure 1.
Sketch (a) of figure 1 illustrates the essential physics of a perforate-over-honeycomb liner. Inside the liner, the
impervious cell walls block any wave motion parallel to the faceplate, leaving only the vertical component free to
propagate. At the first cavity resonance (quarter wavelength), the particle velocity is distributed approximately as 
a)

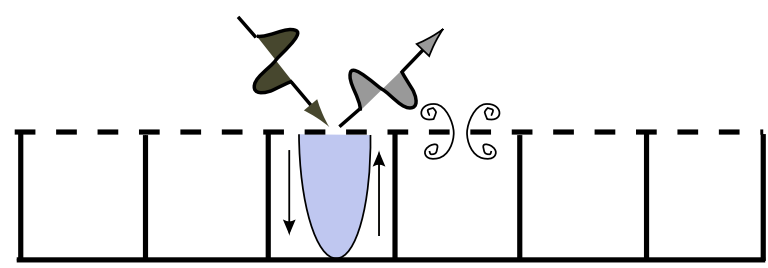

b)

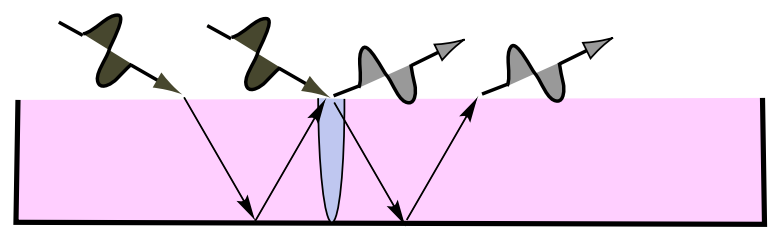

c)

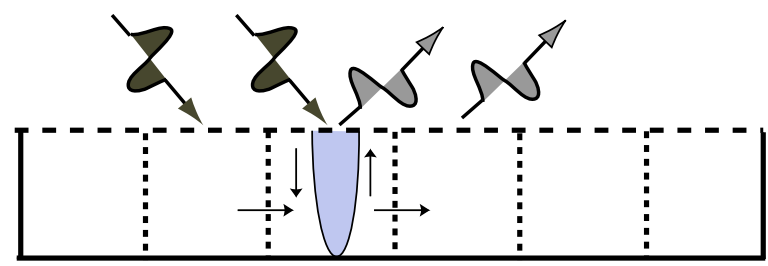

Figure 1. Resonant, bulk and "hybrid" absorbers.

shown by the shaded parabolic region, i.e. zero at the back plate and maximum near the faceplate. Sound absorption is due to viscous and vortical flows generated in and around the faceplate holes. These flows are greatly enhanced at resonance because of the faceplate constriction. As the excitation frequency moves from below resonance to above resonance, the particle velocity anti-resonance plane moves from above the faceplate, through the faceplate and into the cavity. Maximum absorption occurs as this plane moves through the faceplate. This "migration" of the antiresonance plane across the faceplate and into the cavity (and further away from the concentrated faceplate resistance) with increasing frequency, is one way to explain the relatively narrow absorption bandwidth associated with this type absorber. The presence of impervious cell walls tends to accentuate the resonance condition by constraining the oscillatory fluid flow to the faceplate.

Sketch (b) of figure 1 illustrates the essential physics of an extended-reacting, or bulk absorber. There is usually a resonance response for this type of liner also, but it is subdued by two circumstances not present for the locally-reacting liner. First, the resistance to fluid motion is no longer concentrated in the faceplate, but is distributed throughout the bulk absorber. Second, the absence of the cell wall allows fluid motion parallel to the liner faceplate. This distributed dissipation tends to be more viscous controlled because there is no high concentration of particle velocity through the faceplate holes (unless, of course, a porous faceplate is added - a necessity in many practical applications). In contrast with the locally-reacting resonant structure depicted in sketch (a), it is clear that as the excitation frequency increases past resonance, the particle velocity anti-resonance plane (now associated with the vertical component of the wave vector) migrates through the absorber surface and into the bulk material, where the relatively high particle velocity region still encounters resistance, resulting in significant absorption. In summary, the relatively narrow bandwidth absorption of the locally-reacting, resonant absorber is associated with the thin region of resistance through which the particle velocity anti-node migrates as it moves closer to the cavity back plate with increasing frequency. In contrast, the relatively wide absorption bandwidth of the bulk absorber is associated with the distributed resistance that the particle velocity anti-node continues to encounter as it migrates toward the back plate, as the excitation frequency increases past the depth-related resonance.

For applications that require both broadband and tonal noise suppression, the the porous honeycomb liner (porous cell walls) is a "hybrid liner" that shares key features of both the resonant and bulk absorbers. However, this "hybridization" needs to be accomplished in a prescribed manner. Sketch (c) of figure 1 illustrates this possibility by means of a porous cell wall structure. In the study described here, we aspire to treat the structure as a one-dimensional, bulk absorber whose acoustic behavior is described by a characteristic impedance and propagation constant. If such is the case, then the classical analysis ${ }^{1,2}$ for duct propagation with extended-reacting liners becomes available to provide efficient parametric studies to assess the merit of these porous honeycomb structures as duct liners. The more computationally intense numerical propagation models can then be reserved for a final "honing" of a specific duct liner 
design. Thus, the objective of this investigation is to explore the possibility of characterizing cascaded perforates as a "bulk" absorber.

a)

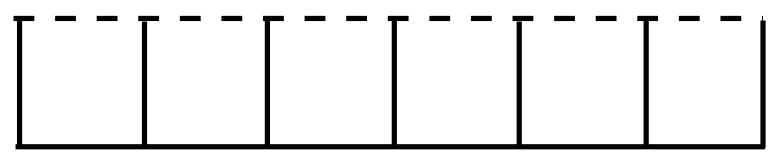

b)

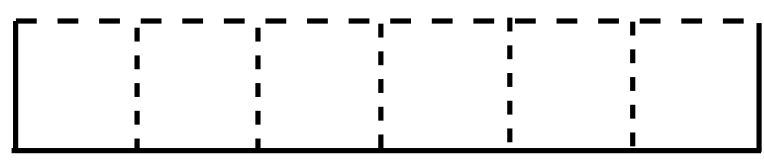

c)

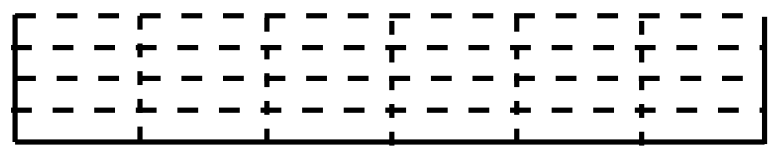

Figure 2. Evolution of conventional to "designer" bulk absorber.

A sufficiently accurate predictive model for the bulk properties of cascaded lumped elements would support the development of "designer" bulk materials. Figure 2 illustrates the evolution of the conventional resonant liner structure (sketch (a)), to a "hybrid" liner (sketch (b)), and finally to a full fledged, 3-D bulk absorber with independently specified bulk properties for each of its three dimensions (sketch (c)).

In a companion paper $^{3}$ for this conference, an experiment is described that validates the hypothesis that a cascade of uniformly-spaced perforates exhibit bulk absorber behavior, at least over the frequency range studied here. There remains the task of developing a predictive model of sufficient accuracy to provide parametric evaluations of bulk liner concepts. This paper describes a first step in this direction.

This investigation is limited to an attempt to predict the "bulk" properties of an $N$-layer cascade of uniformlyspaced perforates. It is based on a two-parameter impedance prediction model (TPIP) for a single perforate, as described in references 2 and 3. The basis of the TPIP model is a semi-empirical characterization of the perforate flow resistance that is manipulated into a non-dimensional form to collapse the flow resistance for the three different perforate porosities $(2.5,5$ and $10 \%)$ to one curve. The semi-empirical constants appearing in this model (laminar flow constant, $a$, and orifice discharge coefficient, $C_{D}$ ) are optimized (least square fit) to all three data sets when plotted versus hole Reynolds number, $R e_{h}$. These optimized values of $a$ and $C_{D}$ are then incorporated into the TPIP model. The TPIP model is then validated against measured impedance spectra for a single perforate-over-cavity configuration, for all three plate porosities and for SPL's of 100, 120, and $140 \mathrm{~dB}$. Finally, predicted impedance spectra from the TPIP model are calculated for uniformly-spaced, 3, 6 and 12-layer perforate cascades. These calculated impedance spectra are taken as simulated data for the two-thickness method to determine the bulk properties. To estimate the efficacy of these "synthesized data," the educed characteristic impedance and propagation constant spectra are then compared with their experimental counterparts, as reported in the companion paper for this conference. ${ }^{3}$

\section{Flow Resistance Model}

$\mathrm{F}$

$$
\frac{R_{f}}{\rho c}=A+B V_{\mathrm{inc}}
$$

where $R_{f}$ is flow resistance, defined as pressure drop, $\Delta p$, across a porous sheet divided by the normal incident, fluid velocity, $V_{\text {inc. }} A$ and $B$ in this equation are treated strictly as empirical constants. In equation 2 below, the $A$ and $B$ are replaced by semi-empirical parameter groups ( $A$-parameter group and $B$-parameter group) that form the basis of a semi-empirical prediction model; ${ }^{4,5}$ i.e.,

$$
\frac{R_{f}}{\rho c}=\frac{a \mu t}{2 \rho c\left(\sigma C_{D}\right) d^{2}}+\frac{\left(k_{i}+k_{e}\right)}{2 c\left(\sigma C_{D}\right)^{2}} V_{\mathrm{inc}}
$$

where the $A$-parameter constituents include perforate hole geometry and fluid properties, and the $B$-parameter accounts 
for the turbulence (nonlinear) contribution. The dissipation mechanisms in this model are fundamentally restricted to a single-hole, in that no interaction between adjacent holes is explicitly included and the hole-to-hole fluid dynamic behavior is assumed invariant. Aside from the geometric constituents of $A$ and $B$, values for the fluid dynamic constituents: the laminar friction coefficient, $a$, the orifice discharge coefficient, $C_{D}$, and the entrance/exit loss coefficients, $k_{i}, k_{e}$, are initially assigned idealized values. These are adjusted for non-ideal circumstances with the aid of flow resistance tests. The basis of the viscous term is found in laminar pipe flow pipe theory, ${ }^{6}$ as evidenced by the explicit appearance of the hole geometry and the fluid viscosity, $\mu$. Hole geometry is notably absent from the second term that deals with dissipation (vortices) exterior to the hole. Initial (or default) values for the four empirical constants $a$, $C_{D}, k_{i}$ and $k_{e}$ are set to the idealized values of $64,0.76,0.5$ and 0.5 , respectively. Of these four, $a$ and $C_{D}$ are subject to adjustment for the limited scope of this investigation. The entrance and exit loss coefficients, $k_{i}, k_{e}$, which enter the model as $k_{i}+k_{e}$, are associated with distortion of flow streamlines on approach to, and exit from, the orifices. Consequently, they are dependent upon hole separation. Adjustments to $k_{i}, k_{e}$ would suggest hole-to-hole interaction, which is assumed negligible for the perforate hole separations studied here.

The no-hole-interaction restriction on the flow resistance model can be emphasized by multiplying equation 2 by perforate porosity and replacing the incident velocity by the in-hole Reynolds number, i.e. $R e_{h}=\left(d V_{\text {inc }}\right) /(v \sigma)$. Equation 2 then reduces to a form where plate porosity is isolated on the left-hand side as a multiplying factor of $R_{f}$. The combination, $\sigma\left(R_{f} /(\rho c)\right)$, is designated as the "reduced flow resistance."

$$
\sigma \frac{R_{f}}{\rho c}=\frac{a \nu}{2 c C_{D} d}\left(\frac{t}{d}\right)+\frac{\left(k_{i}+k_{e}\right)}{2 c C_{D}^{2}}\left(\frac{v}{d}\right) R e_{h}
$$

Another way to describe flow resistance data is by way of the aerodynamic pressure drag coefficient. This coefficient, $C_{p d}$, is defined as $\Delta p / V_{\infty}$, where $\Delta p$ is the pressure drop across the object of interest (e.g., aerodynamic drag on an airfoil) and $\rho V_{\infty}^{2}$ is proportional to the free-stream dynamic pressure; i.e., the aerodynamic $Q$. In the present context, the pressure drag is taken to be the pressure drop, $\Delta p$, across the plate due to the streaming flow through it. The free-stream velocity, $V_{\infty}$, thus becomes the incident velocity on the plate, $V_{\text {inc }}$. From the definition of flow resistance as $\Delta p / V_{\text {inc }}$, equation 3 can be further manipulated to frame the pressure drop, $\Delta p$, across the plate as $C_{p d}=\Delta p /\left(\rho V_{\text {inc }}^{2}\right)$. In this case, the square of porosity appears as a multiplying factor on the left-hand side and permits the hole Reynolds number to appear in its more "natural" setting as an inverse multiplying factor on the viscous term while the turbulence contribution is constant. Multiplying $C_{p d}$ by $\sigma^{2}$ again reduces all three plate porosities to a single curve as shown in equation 4 . The quantity $\sigma^{2} C_{p d}$ is thus called the "reduced pressure drop coefficient." Both equations 3 and 4 are equally valid ways of characterizing the physics of fluid streaming through a thin, perforated plate under the assumption that the only fluid dynamic variable of importance is hole Reynolds number. If fluid compressibility is deemed significant, the functionality would include hole Mach number. For acoustic excitations, this is generally not the case.

$$
\sigma^{2} C_{p d}=\frac{a}{2 C_{D}}\left(\frac{t}{d}\right)\left(\frac{1}{R e_{h}}\right)+\frac{\left(k_{i}+k_{e}\right)}{2 C_{D}^{2}}
$$

\section{Perforate Impedance Model Development}

\section{A. Perforate Test Specimens}

$\mathrm{T}$

HE perforated-plate test specimens were designed to make the viscous term the dominant contributor to resistance at moderate SPL's. To this end, a hole length-to-diameter ratio of 5 was targeted. Four different sets of test specimens (differing in porosity only) were fabricated from type 307 stainless steel. All plates had active area dimensions of $50.8 \times 50.8 \mathrm{~mm}$ and a nominal thickness of $1.27 \mathrm{~mm}$. The nominally circular holes were targeted to a diameter of $0.254 \mathrm{~mm}$, and were implemented by means of laser drilling in a uniform, equilateral triangular pattern over the active areas. Hole spacing was targeted to porosities of $2.5,5$ and $10 \%$ with respective hole spacing of 36, 18, and 9 hole diameters. With the exception of the $2.5 \%$ porosity set, up to 64 plates from a given set could be cascaded with uniform spacing to simulate a one-dimensional "lumped element bulk absorber." Further details of the experimental arrangement for measuring impedance of the test specimens are given in the companion paper. ${ }^{3}$

Figure 3 shows electron-micrograph images of four typical hole geometries. On this scale, "hole circularity" is clearly a relative term. The hole edge roughness was notably greater on the laser beam exit side than on entry side. A representative entry side is shown in the upper left hand image. The remaining three images have edge roughness features as delimited by the concentric circles. These roughness features are likely associated with the hole exit sides. (Entry/exit sides were not documented by the manufacturer.) On these images, hole diameter variability ranges from 

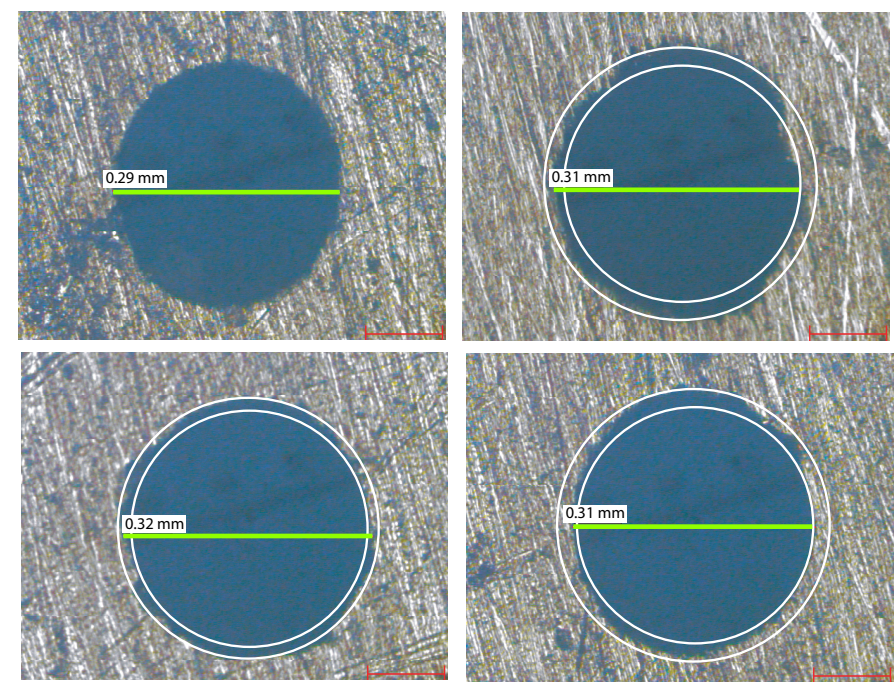

Figure 3. Electron-micrograph images of four typical hole geometries.

nearly zero up to $15 \%$ for the targeted diameter. This is consistent with 24 measurements of hole diameter, taken from similar electron-micrograph images, to obtain a mean hole diameter of $0.304 \mathrm{~mm}$, or about $20 \%$ greater than the targeted value. This mean hole diameter, as derived from electron-micrograph images, was used to correct the targeted (nominal) values. Thus, the nominal porosities of $2.5,5$ and $10 \%$ become $3.6,7.2$ and $14 \%$, respectively. Also, based upon measurements, the nominal plate thickness of $1.27 \mathrm{~mm}$ was corrected to $1.22 \mathrm{~mm}$ and the nominal hole diameter-to-length ratio was corrected from 5 to 4 . These "actual" geometric parameters are used throughout the data reduction procedures and analysis that follow; however, to simplify results presentation, the "tags" of $2.5 \%, 5 \%$ and $10 \%$ are retained in all figures.

It is evident from the images in figure 3 that "hole edge roughness" varies dramatically between what is believed to be the beam entry side (relatively round and smooth edges) and the exit side (relatively out-of-round and ragged). Plate orientation relative to the incident sound was not taken into account, and was assumed random for both flow resistance and acoustic impedance measurements. To a reasonable approximation for tonal excitation, the particle velocity period would be expected to be time-symmetric (i.e., equal time going either direction through a hole). Consequently, hole end-to-end asymmetry would be expected to "average out" insofar as acoustic impedance is concerned, but might not be expected to do so for the flow resistance (i.e., flow resistance is orientation sensitive). However, up to five flow resistance tests conducted on different plates of the same porosity (randomly chosen from the set of 64) failed to demonstrate consistent divergence between the two data sets. Thus, it was concluded that plate orientation (i.e., hole end-to-end asymmetry) has no effect. It would not be surprising, however, to find that hole edge roughness, and other departures from the ideal geometry assumed in the impedance model, have a significant fluid dynamic effect. Thus, adjustments to the classical idealizations embedded in the two-parameter impedance model are to be anticipated.

\section{B. Perforate Flow Resistance Measurements}

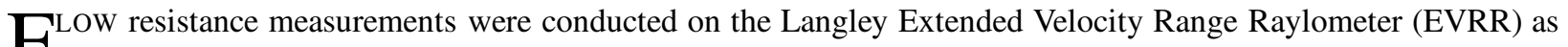
depicted by the sketch of figure 4 . The flow path of the raylometer is similar to that of a blow-down wind tunnel, with a design emphasis on flow quality at the incident side of test sample. Metered air is diffused into a thermally insulated plenum chamber equipped with a turbulence dampening honeycomb mesh and three layers of wire-mesh screen. The flow exits the plenum through a 28:1 contraction into a short section of $51 \mathrm{~mm}$ diameter duct containing a combination pitot-static pressure and total temperature probe. Flow exits from the short duct into a two-piece "test section" duct that holds the test sample and an array of 80 static pressure ports (spaced $6 \mathrm{~mm}$ apart), arranged in a spiraling pattern on either side of the sample (see fig 4). From the test section, the flow is diffused to the atmosphere. The apparatus is designed to accommodate an incident-on-sample velocity range of $1 \mathrm{~mm} / \mathrm{s}$ to $5 \mathrm{~m} / \mathrm{s}$. Eight massflow controllers are used to achieve consistent accuracy over this 5000:1 velocity range. Direct aero-thermodynamic measurements obtained in the short duct, upstream of the sample are used to relate mass flows to average incident velocities on the sample. 


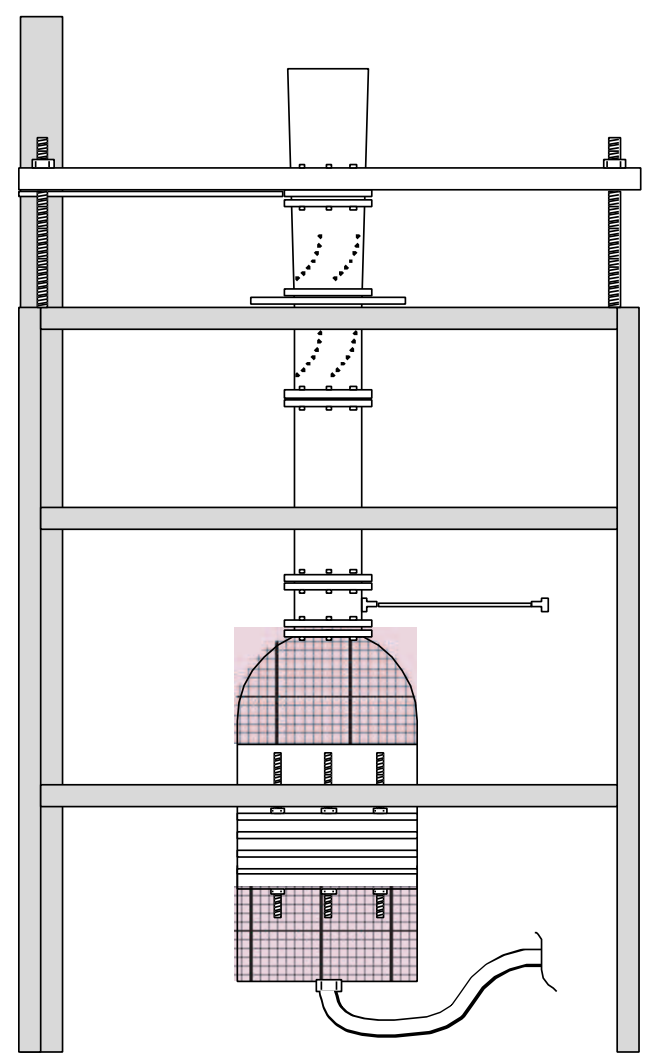

Figure 4. Sketch of Langley Extended Velocity Range Raylometer(EVRR).

The EVRR operation is controlled by a dedicated personal computer. From a programmed sequence of targeted, cross-section averaged incident velocities and the resulting pressure drops across the sample (calculated from an extrapolation of linear regressions to the upstream and downstream static pressure measurements), the flow resistance profile of the sample is automatically determined.

Bias error was addressed in the design of the EVRR as a measurement system using component error tolerances as per manufacture's specifications. Component errors were combined according to a procedure recommended by Coleman. ${ }^{7}$ This cumulative bias error from all subsystems was estimated to be about $1 \%$ of the measured flow resistance. Precision error has not been systematically documented for the EVRR; however, table 1 shows the results for a limited study that are specific to this test. Five randomly chosen plates from each porosity group (2.5, 5, and $10 \%)$ were tested at nominal incident velocities of 200, 1050 and $2000 \mathrm{~mm} / \mathrm{s}$. Minimum and maximum percent deviations from mean values of the respective normalized flow resistances are shown in the last two columns. These results contain both measurement precision error and plate-to-plate variability, without regard to plate orientation. Most of the error shown in table 1 is believed attributable to plate-to-plate variability.

\section{Perforate Flow Resistance Profiles}

EASURED flow resistance profiles (normalized flow resistance versus incident velocity) for the 2.5, 5 and $10 \%$ $\mathbf{1}$ plate porosities are shown in figure 5 along with least square fits of the two-parameter, empirical model as prescribed by equation 1 . To within the graphical resolution, data scatter relative to their respective fits is nil, with the exception of the $2.5 \%$ data set (blue symbols and curve). The single outlier at the incident velocity of $3.8 \mathrm{~m} / \mathrm{s}$ is likely modified due to compressibility associated with high hole velocity (about $106 \mathrm{~m} / \mathrm{s}$ ) for this lowest porosity plate. A second fit (red), with this point excluded, is much more in line with the remaining data points. Some industry practitioners take the measured flow resistance at $105 \mathrm{~cm} / \mathrm{sec}$ as the nominal linear flow resistance, accompanied by a so-called nonlinear factor (NLF) computed as the ratio of flow resistance at $200 \mathrm{~cm} / \mathrm{s}$ to that at $20 \mathrm{~cm} / \mathrm{s}$. In table 2, these quantities are listed for the three perforates (computed from fits on $A$ and $B$, enclosed by parentheses), and from fits using the physics-based parameters, $a$ and $C_{D}$ (without parentheses - to be discussed later). The asterisk indicates values from the $2.5 \%$ data set with the outlier data point included. Excluding the outlier value, there is close 
Table 1. Percent Deviation from Mean (5 Samples)

\begin{tabular}{|c|c|c|c|c|c|c|}
\hline Plate Porosity, $\%$ & Ave Vel, $\mathrm{mm} / \mathrm{s}$ & Ave $R_{f} / \rho c$ & $\operatorname{Min} R_{f} / \rho c$ & $\operatorname{Max} R_{f} / \rho c$ & Min Error, \% & Max Error, \% \\
\hline \multirow{3}{*}{2.5} & 200 & 1.64 & 1.46 & 1.74 & -10.8 & 6.1 \\
& 1050 & 4.40 & 3.79 & 5.04 & -13.9 & 14.4 \\
& 1994 & 7.59 & 6.30 & 8.84 & -17.0 & 16.5 \\
\hline \multirow{3}{*}{5} & 200 & 0.52 & 0.49 & 0.53 & -5.1 & 3.2 \\
& 1051 & 1.13 & 1.04 & 1.18 & -8.1 & 4.2 \\
& 2002 & 1.74 & 1.58 & 1.83 & -9.2 & 4.8 \\
\hline \multirow{3}{*}{10} & 200 & 0.23 & 0.22 & 0.26 & -6.4 & 12.3 \\
& 1051 & 0.38 & 0.36 & 0.44 & -5.6 & 15.1 \\
& 2004 & 0.55 & 0.50 & 0.63 & -9.1 & 12.8 \\
\hline
\end{tabular}

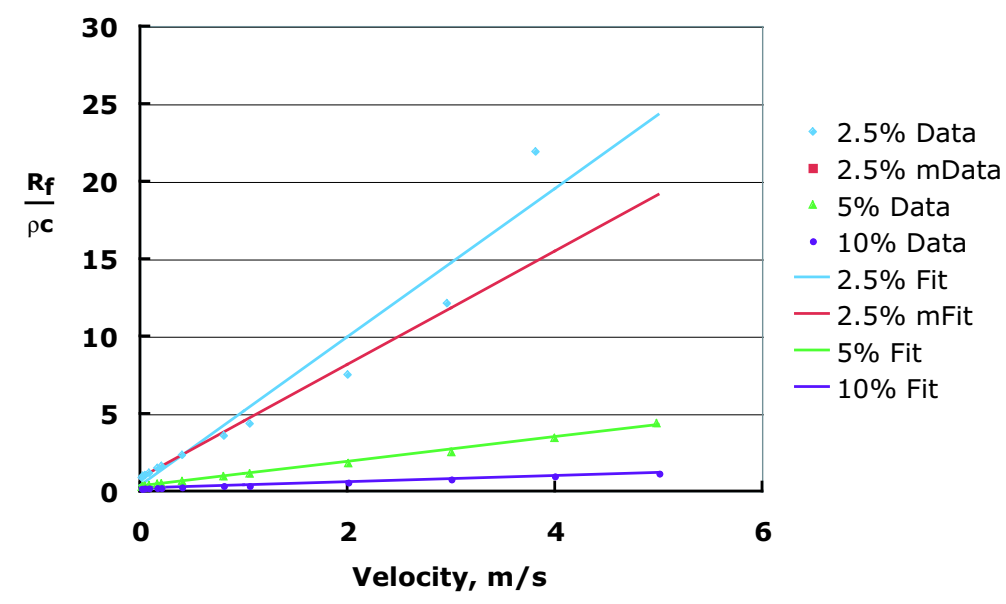

Figure 5. Empirical fits to measured flow resistance profiles.

agreement between the fits on the purely empirical parameters $A$ and $B$ and the on the physics based parameters $a$ and $C_{D}$. However, there is not exact agreement. The small differences apparently reflect the fact that the fit on $C_{D}$ is nonlinear. As is evident, inclusion of the outlier at $3.8 \mathrm{~m} / \mathrm{s}$ distorts both the nominal resistance value and the NLF. The NLF's of 3.8 and 2.7 for the $5 \%$ and $10 \%$ plates, respectively, are to be compared with the most linear of fibermetal products with NLF's ranging from 1.1 to 2.5 . The targeted hole length-to-diameter ratio of 5 (actual value of 4) did not achieve the degree of linearity desired (due, in part, to an actual $C_{D}$ that was less than the idealized value of 0.76 by about 30\%). Consequently, the goal of achieving linear acoustic resistance values well within the measurement "comfort range" of the Langley NIT capability was only marginally achieved.

Table 2. Plate porosity and flow resistance estimates from fits to equation 1

\begin{tabular}{|c|c|c|}
\hline Plate Porosity & $\frac{R_{f}}{\rho c}$ at $105 \mathrm{~cm} / \mathrm{s}$ & Nonlinear Factor \\
\hline $2.5 \%$ & $4.3(5.4)^{*}$ & $5.3(7.5)^{*}$ \\
\hline $5 \%$ & $1.3(1.2)$ & $3.8(3.9)$ \\
\hline $10 \%$ & $0.4(0.4)$ & $2.7(2.5)$ \\
\hline
\end{tabular}

A difficulty arises when the simple empirical fits, depicted in figure 5, are used for estimating the limiting extent of the nominal linear resistance region for flow resistance data described on the basis of a two-parameter model. This is evident at the low incident velocity range (less than about $0.2 \mathrm{~m} / \mathrm{s}$ ) in figure 5, where an attempt is made to define the transition from viscous to turbulence-controlled resistance by means of the relatively high density of incident 


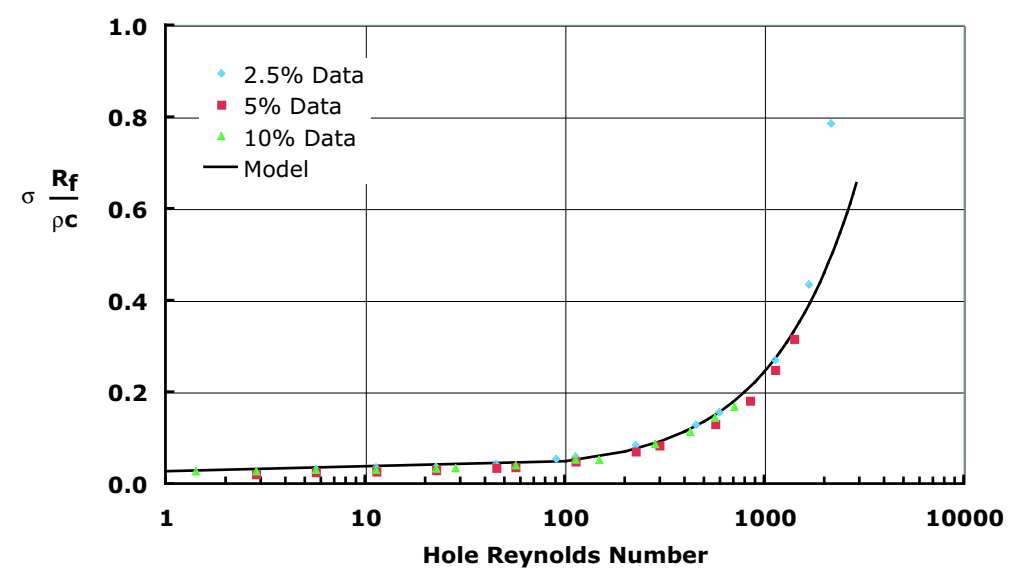

Figure 6. Reduced flow resistance profile.

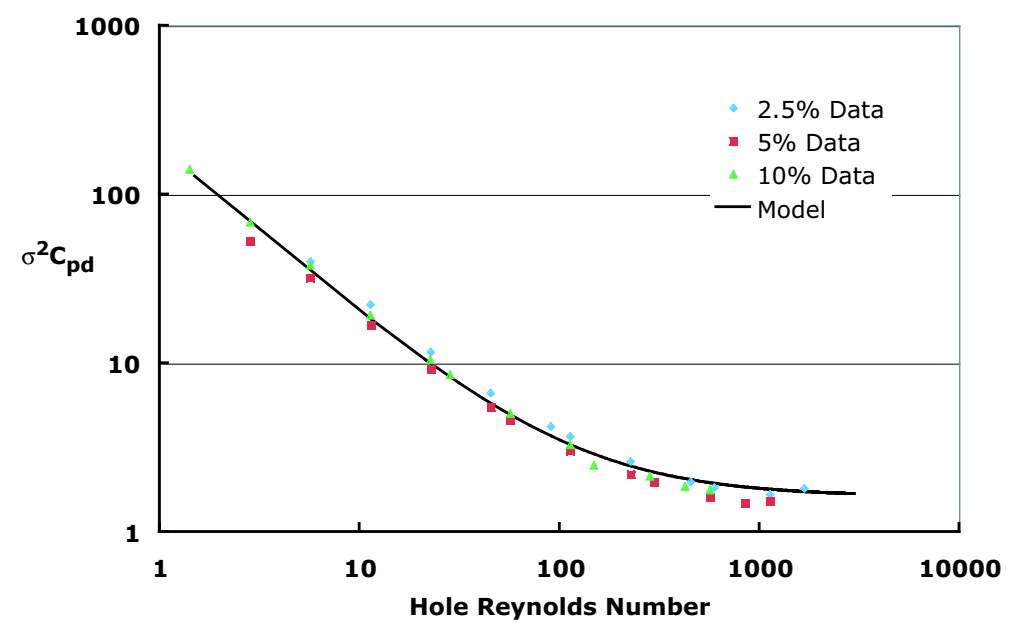

Figure 7. Reduced pressure drop coefficient profile.

velocities. On a linear scale, these more closely spaced velocity points provide little insight into the nature of this transition region. In figure 6, three steps are taken to help clarify the contributions from the viscous and turbulence terms in equation 1. First, a log scale is used to bring focus to the low incident velocity range. Second, the purely empirical parameters $(A$ and $B$ ) are replaced by their semi-empirical counterparts to allow the plate porosity to be isolated as a multiplying factor on the flow resistance (as per equation 2). Finally, the average incident velocity on the sample has been replaced by an average in-hole Reynolds number. The efficacy of this procedure is that it allows all three plate porosities to be collapsed to a cluster around one curve. This collapsed data representation is called the "reduced flow resistance." It is a result of having chosen the holes in all three plates to have the same hole length-todiameter ratio. The above procedure is pursued one step further in figure 7, wherein flow resistance has been replaced by a "reduced pressure drop" coefficient across the sample as per equation 3. Again, the data from all three plates are expected to cluster around a single curve.

Figure 6 shows reduced flow resistance, $\sigma\left(R_{f} /(\rho c)\right)$, plotted versus hole Reynolds number, along with a composite, least squares fit to the combined three data sets (solid curve). Note that the composite fit excludes the hole Reynolds number value at 2100 because of the conjectured compressibility effect discussed earlier (the $3.8 \mathrm{~m} / \mathrm{s}$ velocity point for the $2.5 \%$ data in figure 5). The "stretching out" of the low range Reynolds numbers shows that the reduced flow resistance suffers relatively little nonlinearity for hole Reynolds numbers less than about 100 as it takes about six doublings of Reynolds number (from 1.5 to 96) to double the reduced resistance. Above 100, transition to the 
turbulence-dominated $B$-term (see equation 1) begins. In this region, a single doubling from 500 to 1000 generates a 2.5 -fold increase in reduced flow resistance. Unlike the empirical fits of figure 5, the composite fit of figure 6 is performed on the laminar friction and the discharge coefficients $\left(a\right.$ and $\left.C_{D}\right)$, the fluid dynamic constituents of the parameter groups $\left(A\right.$ and $B$ ). While this fit is linear with respect to $a$, it is not linear with respect to $C_{D}$. Furthermore, the $a$ and $C_{D}$ cannot be adjusted independently. (This is not the case for the empirical fit on $A$ and $B$.) This means there is no unique optimum for $a$ and $C_{D}$. Although "engineering judgment" can be employed to prioritize, or weight, optimal choices for $a$ and $C_{D}$, these options were not pursued here. Instead, a straightforward mathematical fitting routine was used to calculate "optimum" values. Consequently, no physical meaning should be attached to the values computed in this manner. The least squares fit optimum values for $a$ and $C_{D}$, using this model, were determined to be 51 and 0.58 , respectively. One advantage of employing this form of data analysis is to achieve a single composite model that "averages out" the nuances in the three data sets (as well as any plate-to-plate variability due to hole-to-hole interaction). It does not take into account, however, the plate-to-plate variability as presented in table 1.

Figure 7 shows a log-log plot of the reduced pressure drop coefficient data, together with the corresponding model fit, as developed in equation 4. Again, the data collapse is judged to be very good to within the limits of measurement error and variability of the hole fabrication process. The model fit to the composite data sets produces optimum values of 50 and 0.56 for $a$ and $C_{D}$, respectively. In this model fit, notwithstanding the removal of the outlier at 2100, the next highest hole-Reynolds number for the $2.5 \%$ data (blue symbols) appears to bias the asymptotic (or $B$-term dominated) part of the model fit to higher values of the reduced pressure drop coefficient. This is borne out by the fact that $C_{D}$ and $a$ optimum values of 50 and 0.56 for this model fit are 2 and $3.5 \%$ smaller than their respective counterparts, respectively, for the reduced flow resistance model fit. Sensitivity studies with the TPIP model determined that these differences did not make a significant impact on the TPIP model comparison with measured impedance spectra. Therefore, the choice of $a_{\mathrm{opt}}=50$ and $C_{D, \mathrm{opt}}=0.56$ were adopted in the TPIP model, mainly because the linear region fit for the reduced pressure drop coefficient seems to have somewhat less bias than did the reduced flow resistance model fit. These minor differences in optimum values for $a_{\mathrm{opt}}$ and $C_{D, \text { rmopt }}$ are believed to reflect the differences in the two non-dimensional characterizations of the flow resistance as expressed in equations 3 and 4; i.e., direct proportionality in one case and inverse proportionality in the other case. These tend to emphasize different aspects of the pressure drop across a perforate subjected to normally incident flow. The arguments presented here are for the purpose of pointing out the potential value in providing both viewpoints (i.e., reduced flow resistance and reduced pressure drop coefficient) for interpreting the fluid physics of laminar and turbulent flow through thin perforates.

\section{Two-Parameter Impedance Prediction (TPIP) Model}

T

HE frequency-domain impedance prediction model (TPIP) is described by $\mathrm{Kraft}^{4}$ and Motsinger. ${ }^{5}$ Modified versions of this generic, semi-empirical model have been used by industry for years to design liners for frequencies up to about $10 \mathrm{kHz}$. A modified version of TPIP is used herein. This version uses the optimized values of the laminar flow friction factor, $a_{\mathrm{opt}}$, and hole discharge coefficient, $C_{D \text {,opt }}$ as computed from the composite fit to the reduced pressure drop coefficient model for the perforated plates (equation 4), as discussed previously. These values for $a_{\text {opt }}$ and $C_{D, \text { opt }}$ are taken to be 50 and 0.56 , respectively (as compared to the classical values 64 and 0.76 ). A key assumption in the TPIP model is the equating of flow resistance from equation 1 to the acoustic resistance of the perforate. This results in the replacement of $V_{\text {inc }}$ by the RMS acoustic particle velocity, $V_{\text {rms }}$ (due acoustic excitation alone in the absence of grazing or bias flow), through the perforate. The physical basis for this assumption is that the plate is sufficiently thin (relative to the acoustic wavelength), and the time scale of particle velocity change is sufficiently small, that it can be regarded as quasi-steady. It is not at all clear that the assumption is warranted when vorticity-generated dissipation becomes a significant contributor. By making the perforate hole $t / d$ sufficiently high, this source of nonlinearity can be masked, to some extent, by the viscous contribution, and thus the above assumption becomes less of an issue. Mass reactance is included in the perforate model in the usual way. ${ }^{4,5}$ Several variations on the classical end corrections were considered to account for the added mass at the hole exits; however, the classical end correction of $\varepsilon=8 d /(3 \pi)$ was chosen as providing a generally reasonable fit to the data. Only tone-at-a-time impedance spectra are computed for the $2.5,5$ and $10 \%$ perforates over a $49.5 \mathrm{~mm}$ depth backing cavity, for incident SPL's of 100,120 and $140 \mathrm{~dB}$. The backing cavity is modeled both as a purely reactive chamber, $-\cot \left(\omega L_{\mathrm{cav}} / c\right)$, or as a cavity with a high backplate impedance, $\zeta_{\text {ter }}$, to account for an observed "spill-out" effect due to an anti-resonance at $3500 \mathrm{~Hz}$. Based on evaluations with multiple choices of impedance, this impedance was chosen to be $(160 \pi / \omega) \cdot 10^{3}$ $\rho c(80 \rho c$ at $1000 \mathrm{~Hz})$. The TPIP model was implemented in a Mathematica code for which the relevant equations are as follows: 
Perforate resistance parameters:

$$
A=\frac{a_{\mathrm{opt}} \mu t}{2 \rho c\left(\sigma C_{D, \mathrm{opt}}\right) d^{2}} ; \quad B=\frac{\left(k_{i}+k_{e}\right)}{2 c\left(\sigma C_{D, \mathrm{opt}}\right)^{2}}
$$

Perforate mass reactance:

$$
\chi_{m}=k\left(\frac{t+\varepsilon d}{\sigma C_{D, \mathrm{opt}}}\right)
$$

The nonlinear response of the perforate requires the incident acoustic pressure, $p_{\text {rms }}$ to be included as a functional dependence to calculate the acoustic RMS velocity through the perforate. An iterative loop is required to converge the calculated impedance to a value consistent with the particle velocity generated by the incident SPL at a given excitation frequency, $\omega$. It is assumed $p_{\text {rms }}$ is known. The iterative loop is as follows:

1. Set $V_{\mathrm{rms}}=0$

2. Calculate the estimated impedance $\left(\zeta_{e}\right)$ using equation 7 if the back plate is assumed rigid $\left(\zeta_{\text {ter }}=\infty\right)$, or using equation 8 if the back plate is assumed non-rigid $\left(\zeta_{\text {ter }}=80 \cdot 10^{3} / f\right)$

3. Update $V_{\mathrm{rms}}$ using $V_{\mathrm{rms}}=p_{\mathrm{rms}} / \zeta_{e}$

4. Iterate on steps 2 and 3 above until convergence is reached.

$$
\begin{gathered}
\zeta_{e}=A+B V_{\mathrm{rms}}+I\left(\chi_{m}-\cot \left(k L_{\mathrm{cav}}\right)\right) \\
\zeta_{e}=A+\operatorname{Real}\left[\frac{\zeta_{\mathrm{ter}}+I \tan \left(k L_{\mathrm{cav}}\right)}{1+I \zeta_{\mathrm{ter}} \tan \left(k L_{\mathrm{cav}}\right)}\right]+B V_{\mathrm{rms}}+I\left(\chi_{m}+\operatorname{Imaginary}\left[\frac{\zeta_{\text {ter }}+I \tan \left(k L_{\mathrm{cav}}\right)}{1+I \zeta_{\mathrm{ter}} \tan \left(k L_{\mathrm{cav}}\right)}\right]\right)
\end{gathered}
$$

For the TPIP model validation (prior to $N$-layer impedance prediction), normal incidence impedance measurements at incident SPL's of 100, 120 and $140 \mathrm{~dB}$ were obtained on all three plate porosities over a cavity depth of $49.5 \mathrm{~mm}$.

\section{E. Comparisons of TPIP Model with Measured Impedance Spectra}

To provide a baseline for the predictive capability of the the modified TPIP model (using optimized values $a_{\text {opt }}$ and

$1 C_{D, \text { opt }}$ ), it is of interest to first compare measured and predicted impedance spectra using the classical (or default) values of 64 and 0.76 for $a$ and $C_{D}$, respectively. Figures 8 and 9 (normalized resistance and reactance, respectively) show this comparison for the $10 \%$ perforate (the most linear of the three tested), for the three incident sound pressure levels (SPL's) of 100, 120 and $140 \mathrm{~dB}$ (blue, red and green, respectively) with tone-at-a-time excitation and with no cavity absorption. With increasing SPL, the predicted resistance spectra begin with a flat spectrum of about $0.18 \rho c$ (blue curve) for the $100 \mathrm{~dB}$ case. For the $120 \mathrm{~dB}$ case (red curve), a resonance-related resistance peak is starting to emerge in the vicinity of $1300 \mathrm{~Hz}$, the zero crossing of the reactance in figure 9 . At $140 \mathrm{~dB}$ (green curve), there is a pronounced, broad resonance-related peak that is about $100 \%$ greater $(0.36 \rho c)$ than the uniform spectrum for the $100 \mathrm{~dB}$ excitation. Before discussing the variance between predicted and measured resistance spectra, it is worth noting the close agreement between predicted and measured reactance spectra of figure 9. For all incident SPL's, predicted and measured reactance spectra cluster together in excellent agreement. This is as expected for this $-\cot \left(k L_{\mathrm{cav}}\right)$ model of cavity reactance and the classical mass reactance model of the perforate (equation 6). It is surprising, however, that there is no detectable shift of the measured reactance spectra for the $140 \mathrm{~dB}$ case due to loss of attached mass at this excitation level. This is true in spite of the fact that the $140 \mathrm{~dB}$ excitation produces a significant nonlinear resistance peak at the system resonance that, for the data trend, is about $50 \mathrm{~Hz}$ less than the predicted peak for this case.

The default TPIP model under-predicts the resistance spectra across the entire frequency range. The underprediction is greatest for the resonance-related peaks and the upper end of the frequency range, where a monotonic increase in measured resistance produces a diverging trend when compared with the predicted spectra. Later, it is shown that the optimized $a$ and $C_{D}$, from previously discussed flow resistance measurements, bring the predicted resistance spectra into much better agreement with the measured spectra for frequencies up through resonance; however, the "contaminating" effect of the anti-resonance is not included in the TPIP as employed in the present discussion. The anti-resonance is a concern because in the $N$-layer cascade application, anti-resonances are present in the measurement frequency range (500 to $3000 \mathrm{~Hz}$ ) because of the increased cascade length relative to the $49.5 \mathrm{~mm}$ cavity depth of interest. 


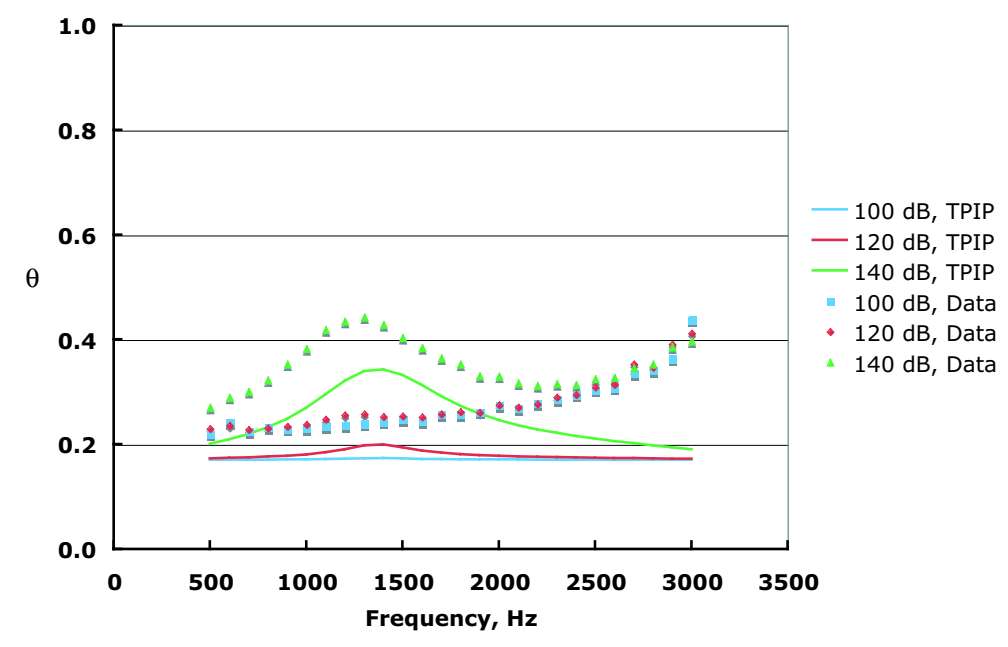

Figure 8. Normalized resistance for $10 \%$ perforate $\left(a=64, C_{D}=0.76, \zeta_{\text {ter }}=\infty\right)$.

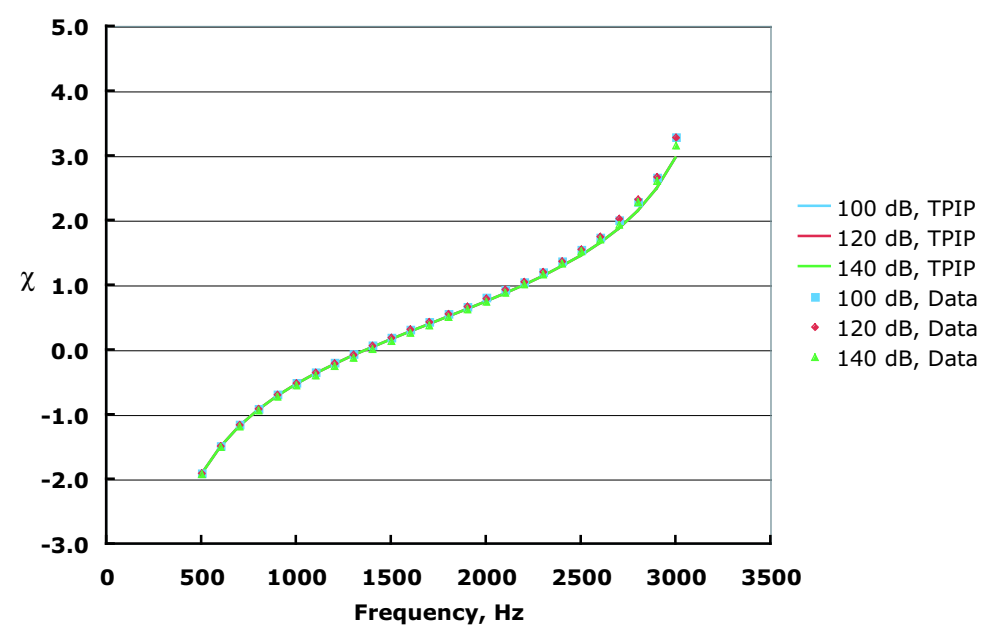

Figure 9. Normalized reactance for $10 \%$ perforate $\left(a=64, C_{D}=0.76, \zeta_{\text {ter }}=\infty\right)$.

The monotonic increase in resistance toward $3000 \mathrm{~Hz}$ is, in part, believed due to an anti-resonance at $3500 \mathrm{~Hz}$. Experimentally, such anti-resonances manifest themselves in the resistance spectrum as resistance spikes (of several tens of $\rho c$ ) that are isolated to a narrow frequency range of $50 \mathrm{~Hz}$ or less, and are quite beyond the measurement capability of the Langley NIT. The spikes are presumably associated with small amounts of acoustic absorption due to viscous and thermal dissipation at the cavity walls. Typically, these classical absorption mechanisms are sufficient to estimate the anti-resonance, resistance bandwidth in a qualitative manner. From the trends evident in figure 8, it might be surmised that this effect is "spilling out" to several hundred Hertz from the anti-resonance frequency, to affect the relatively low resistance spectra of the perforate-cavity system down to at least $2000 \mathrm{~Hz}$. Such a "spill-out" would be consistent with a cavity absorption much greater than that predicted from classical absorption. Arguably then, there is no physical basis for this anomalous cavity absorption.

It is believed that a pressure field resolution issue comes into play with the $6 \mathrm{~mm}$-diameter microphones used to measure the transfer function in the standing wave field. As the excitation frequency increases past the system resonance toward the upper limit of $3000 \mathrm{~Hz}$, the standing wave ratio (SWR) increases (mass reactance increases). The wavelength continuously decreases, making the sampling area (microphone diameter) greater relative to wavelength over which the pressure and phase are averaged. At a microphone separation of $25 \mathrm{~mm}$, the standing wave field "curvature" presented to the two microphones may well be quite different, tending to produce a systematic error. In 


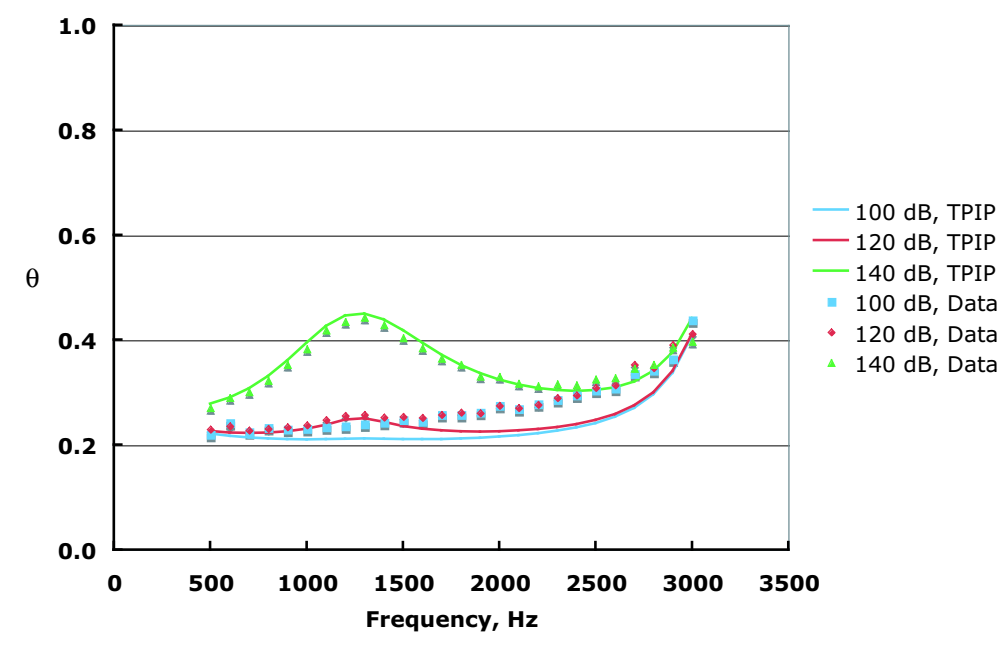

Figure 10. Normalized resistance for $10 \%$ perforate $\left(a_{\mathrm{opt}}=50, C_{D, \mathrm{opt}}=0.56, \zeta_{\mathrm{ter}}=\left(80 \cdot 10^{3} / f\right) \rho c\right)$.

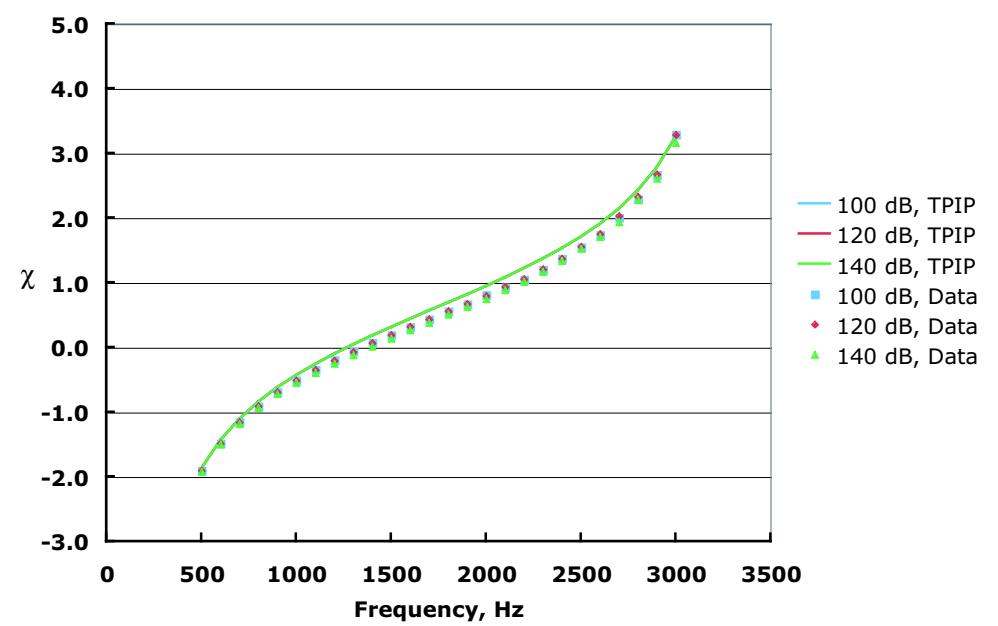

Figure 11. Normalized reactance for $10 \%$ perforate $\left(a_{\mathrm{opt}}=50, C_{D, \mathrm{opt}}=0.56, \zeta_{\mathrm{ter}}=\left(80 \cdot 10^{3} / f\right) \rho c\right)$.

this circumstance, the measurement system may conceivably generate what appears to be an anti-resistance "spill-out" of up to $0.2 \rho c$. As an expediency in what follows, the cavity back plate is imputed a resistance that mimics this effect.

Figure 10 shows a comparison of measured and predicted impedance spectra for the $10 \%$ perforate and with an imputed cavity back plate resistance of $\left(80 \cdot 10^{3} / f\right) \rho c$. The cavity model switches form equation 7 to equation 8 . The TPIP was modified by setting the laminar flow friction constant, $a$ and discharge coefficient, $C_{D}$, to the optimized values of 50 and 0.56 , respectively (from the least squares fit to the reduced pressure drop coefficient data). These model "adjustments" allow reasonably good agreement to be attained between predicted and measured resistance spectra, especially for the $140 \mathrm{~dB}$ case. For the $120 \mathrm{~dB}$ case, measured and predicted resistance spectra are in excellent agreement up the predicted resonance peak. Thereafter, they diverge significantly up to about $2500 \mathrm{~Hz}$, where the antiresonance correction brings convergence. For the $100 \mathrm{~dB}$ case, measured and predicted spectra agree only at the lowest frequencies and diverge for frequencies up to about $2500 \mathrm{~Hz}$ where again convergence begins. It is believed that, were it not for the pseudo-anti-resonance effect, the modified TPIP would exhibit good agreement with measurement throughout the frequency range.

Figure 11 shows a comparison of measured and predicted reactance spectra for the modified TPIP model as described above. The measured and predicted reactance spectra are seen to be in very good agreement, with the measured data trends shifting to slightly higher frequencies to produce a higher zero-crossing by about $50 \mathrm{~Hz}$. The predicted 


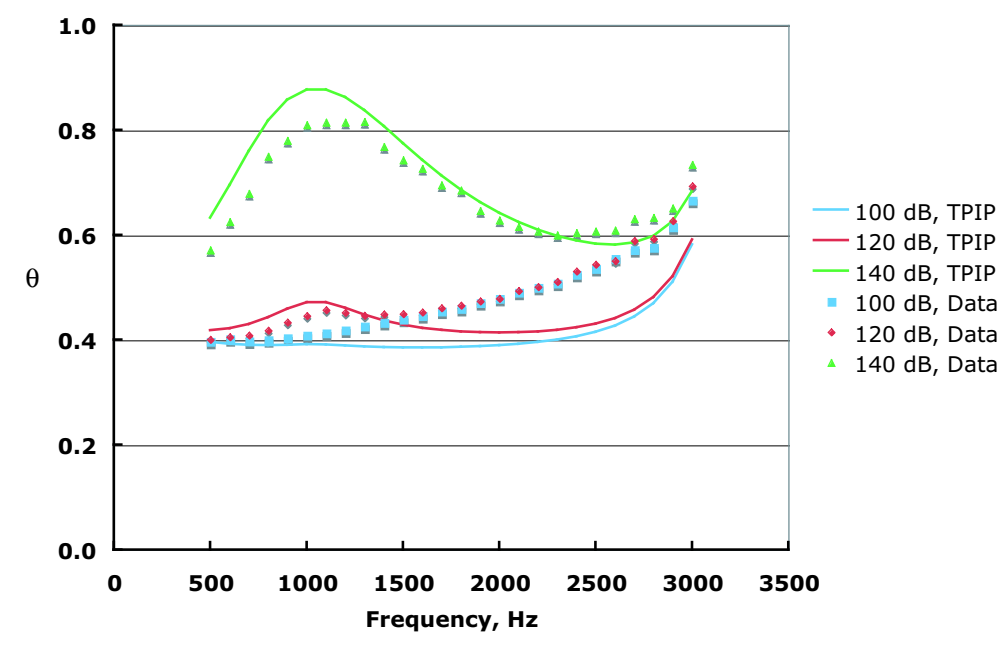

Figure 12. Normalized resistance for $5 \%$ perforate $\left(a_{\mathrm{opt}}=50, C_{D, \mathrm{opt}}=0.56, \zeta_{\mathrm{ter}}=\left(80 \cdot 10^{3} / f\right) \rho c\right)$.

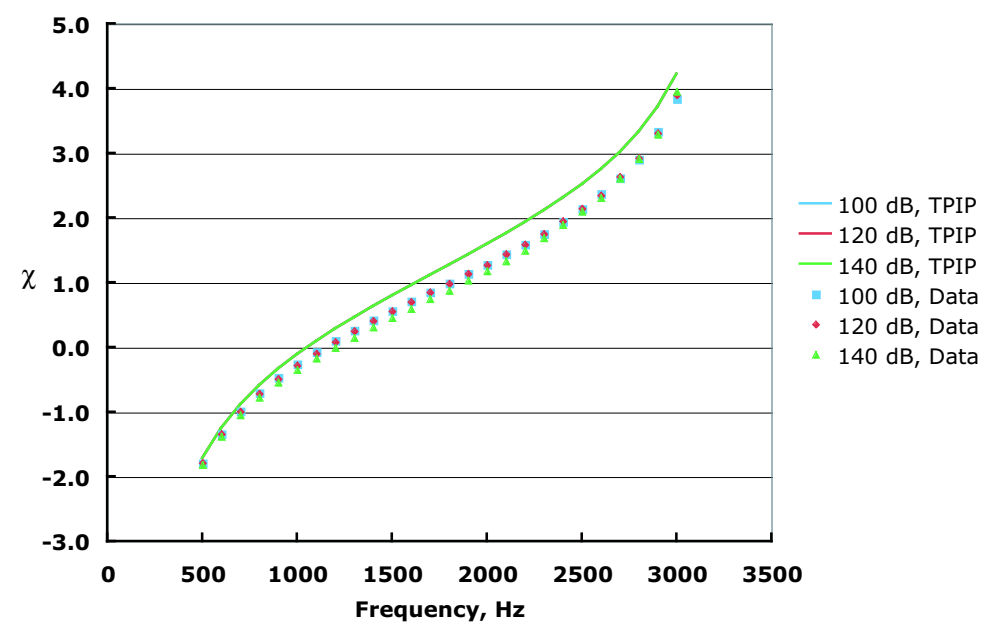

Figure 13. Normalized reactance for $5 \%$ perforate $\left(a_{\mathrm{opt}}=50, C_{D, \mathrm{opt}}=0.56, \zeta_{\mathrm{ter}}=\left(80 \cdot 10^{3} / f\right) \rho c\right)$.

zero-crossing aligns almost perfectly with the associated nonlinear resistance peaks in figure 10. Apparently, the "slightly absorbing" cavity model is also a slight improvement over the $-\cot \left(k L_{\text {cav }}\right)$ model. However, for these relatively high $t / d$ perforates, the effect is minimal.

Figure 12 shows a comparison of measured and predicted resistance spectra for the 5\% perforate. The modified TPIP model with the "slightly absorbing" cavity is used for the prediction. At $140 \mathrm{~dB}$, the model over-predicts by nearly $0.1 \rho c$ up to the resonance related peak at $1100 \mathrm{~Hz}$. Above $1100 \mathrm{~Hz}$, the over-prediction decreases up to a cross-over frequency at $2300 \mathrm{~Hz}$, where the model begins to under-predict. It reaches a maximum under-prediction of about $0.05 \rho c$ at $2700 \mathrm{~Hz}$, and then starts to converge with the data trend. For the 100 and $120 \mathrm{~dB}$ cases, predicted and measured spectra are in good agreement at low frequencies with the $120 \mathrm{~dB}$ case being over-predicted past resonance to about $1500 \mathrm{~Hz}$, but then beginning to diverge significantly from the predicted trend. The $100 \mathrm{~dB}$ predicted and measured spectra are in excellent agreement at low frequencies, but begin to diverge at $1100 \mathrm{~Hz}$.

Figure 13 shows comparisons of measured and predicted reactance spectra for the $5 \%$ perforate. There is significant spread between predicted and measured reactance trends for all SPL's, with the $140 \mathrm{~dB}$ data trend being slightly to the right of the other data trends. All data trends are right shifted with respect to the predicted trends. This is what would be expected for a reduction in mass reactance with increasing SPL; however, the predicted zero-crossing seems to align perfectly with the associated nonlinear resistance peaks in figure 12. 


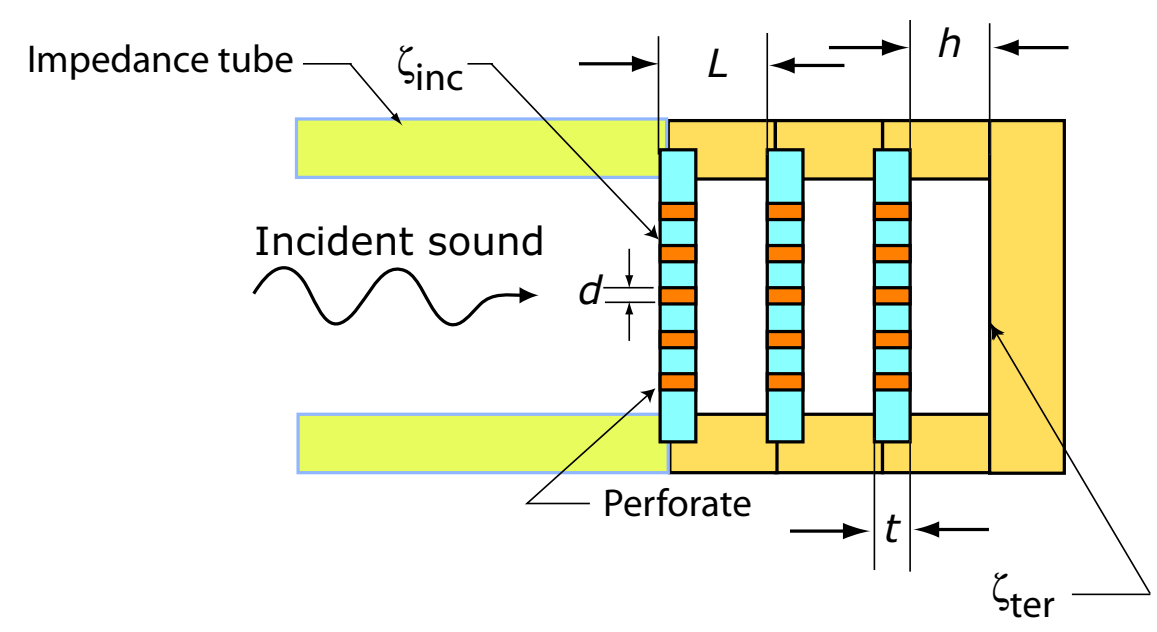

Figure 14. 3-layer cascade of perforate/air spaces attached to a normal incidence impedance tube, $h=5.51 \mathrm{~mm}, t=1.22 \mathbf{m m}, L=6.73 \mathbf{m m}$.

Measured and predicted impedance spectra were also obtained and compared for the $2.5 \%$ plate. The comparison showed further degradation of model versus data relative to that for the 5\% plate, particularly for the lower SPL measurements, at least for the optimum choices $a$ and $C_{D}$ used. This was not unexpected, because the $2.5 \%$ plate exhibited nonlinear response at lower excitation SPL than did the higher porosities. It was found that readjustment of $a$ and $C_{D}$, as well as the backing plate imputed impedance, could bring the 2.5\% TPIP model prediction into better agreement with measured spectra. However, the goal of this investigation is to demonstrate the efficacy of using a first-principles impedance prediction model to characterize "bulk properties" of a cascaded lumped element structure. Any additional ad-hoc "tweeking" of $a$ and $C_{D}$ to improve compliance of the TPIP model with measured spectra was deemed not within the purpose of this investigation.

A key part of this investigation was to evaluate the effects of combining perforates into a cascaded configuration such that "bulk" properties can be educed. For convenience, it was decided that only one of the porosities would be used for this detailed study. It is evident from the comparisons above that model and measured data are in best agreement for the $10 \%$ plate and in worst agreement for the $2.5 \%$ plate. The $10 \%$ porosity was eliminated because of the following two concerns. First, there was a concern that the propagation constant would not be comparable to that achieved with typical, "real" bulk materials. Second, the nonlinear effects observed at the highest SPL (140 dB) are clearly more dominant for the $5 \%$ porous plate. It was thus decided to use the $5 \%$ plate for $N$-layer calculations to provide cascade surface impedance spectra for educing "bulk properties."

\section{Impedance Calculation for Cascaded Perforates}

$\mathrm{T}$ HE previous section assessed the ability of the modified TPIP model to predict impedance spectra for individual perforates over a cavity of depth $49.5 \mathrm{~mm}$. The model provided very good accuracy for the $10 \%$ plate resistance, especially for the $140 \mathrm{~dB}$ case. For the 120 and $100 \mathrm{~dB}$ cases, predicted resistance was in good agreement up through resonance, but, divergences between predictions and data trends, believed due to the anti-resonance effect, were noted at higher frequencies. Prediction accuracy further deteriorated for the 5\% plate for all SPL levels, relative to the $10 \%$ plate, but was still reasonably good, except that the anti-resonance divergence was even more severe. Predicted reactance spectra were in very good agreement with data trends. For the 5\% plate, the reactance data trend shifted about 50 to $100 \mathrm{~Hz}$ higher relative to the prediction (the mass reactance model is SPL insensitive), presumably because mass reactance was diminished by the higher hole velocities in the 5\% plate as was expected. It is believed that, had it not been for the anti-resonance effect, the TPIP model would have done much better at the lower SPL's of 100 and $120 \mathrm{~dB}$. The anti-resonance effect issue notwithstanding, the TPIP was employed to predict the normal incidence impedance of cascaded sets of perforates consisting of 3,6 and 12 layers. These predicted impedance spectra were also compared with measurements obtained in an impedance tube and described in the companion paper.

A sketch of a 3-layer cascade is illustrated in figure 14. Each layer consists of an air space and a plate of 5\% porosity. The air space separations are set to $5.51 \mathrm{~mm}(0.217 \mathrm{in}$ ). The plate is countersunk (with a tight, sealed fit) into a plastic frame, forming the air space to a depth such that the combined air space and plate thickness is $6.73 \mathrm{~mm}$. The 


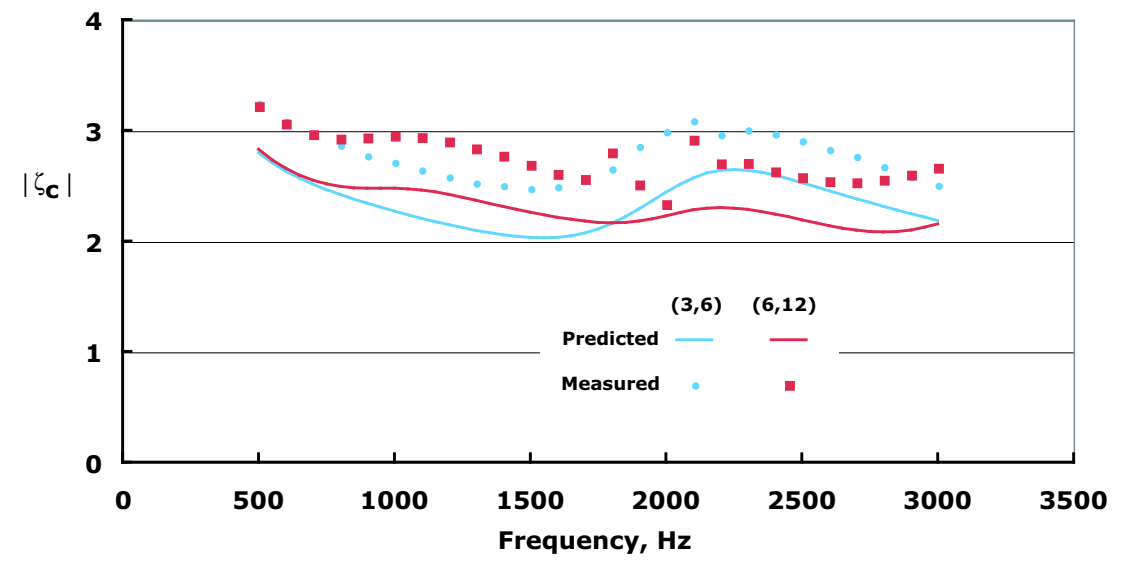

Figure 15. Characteristic impedance modulus for $(3,6)$ and $(6,12)$-layer pairs of $5 \%$ perforates, SPL of $120 \mathrm{~dB}$.

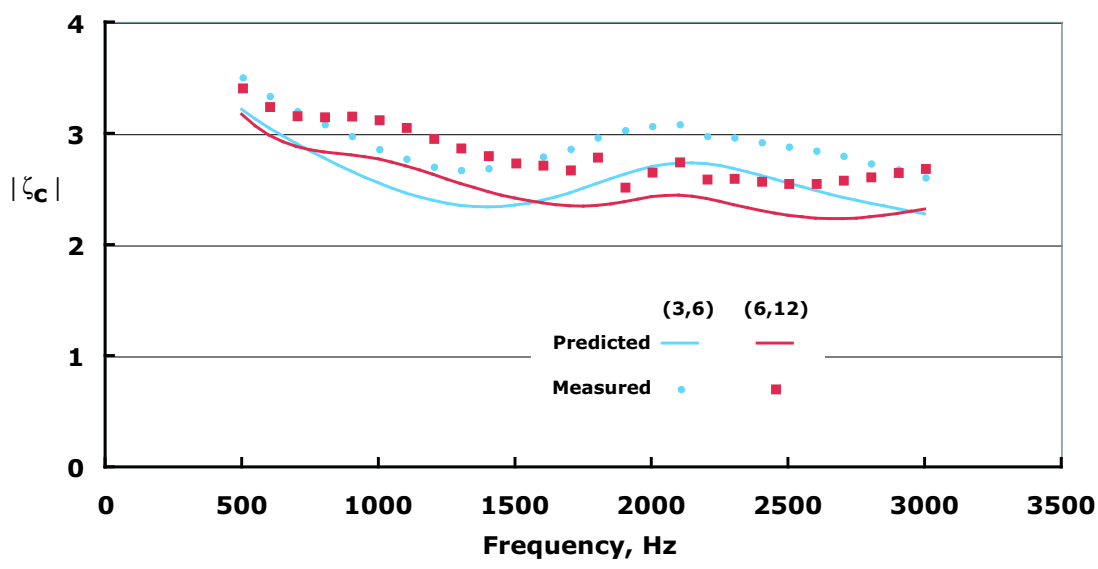

Figure 16. Characteristic impedance modulus for $(3,6)$ and $(6,12)$-layer pairs of $5 \%$ perforates, SPL of $140 \mathrm{~dB}$.

cascade is then clamped tightly onto the impedance tube and tone-at-a-time impedance measurements are conducted as described in the companion paper. ${ }^{3}$ It is important to note that cascade layer length, $L$ (figure 14), is taken to be the "effective" air-space length in the transmission matrix that describes propagation through the air space. This is a necessary "adjustment" for consistency with the "thin" plate assumption, whereby wave propagation effects inside the plate are assumed absent. However, the added length amounts to about $22 \%$.

The TPIP impedance model for the perforate is used, in its modified form, to predict the normal incidence impedance of the $N$-layer cascades; however, the artifice of setting the back plate impedance to $\left(80 \cdot 10^{3} / f\right) \rho c$ is not employed. The use of this back-plate impedance produces meaningless results (a further indication that this "imputed" impedance is not real). Instead, the back plate impedance is set to $2.5 \cdot 10^{6} \rho c$, which, for practical purposes, is infinite. The TPIP model for the perforate was rearranged into a two-port transfer matrix for incorporation into a transmission matrix format. ${ }^{8}$ The cascade impedance is calculated using two-port transfer impedances for perforates and air spaces. The calculation marches sequentially from an initially assumed back-plate pressure to the incident side of the faceplate, to calculate an estimate of the normal incidence impedance. From this estimated impedance, the incident pressure is calculated and compared with the measured incident pressure. With this information, the back plate pressure is adjusted in the appropriate direction until a tolerance is met between estimated and measured incident pressures. In this manner, the perforate nonlinearity is included in the impedance spectra predictions for the $N$-layer cascades.

Computations were conducted for 3, 6 and 12-layer cascades, for incident SPL's of 120 and $140 \mathrm{~dB}$. For the sake of brevity, graphical comparisons of predicted and measured impedance for these 12 cases are not presented. Somewhat 


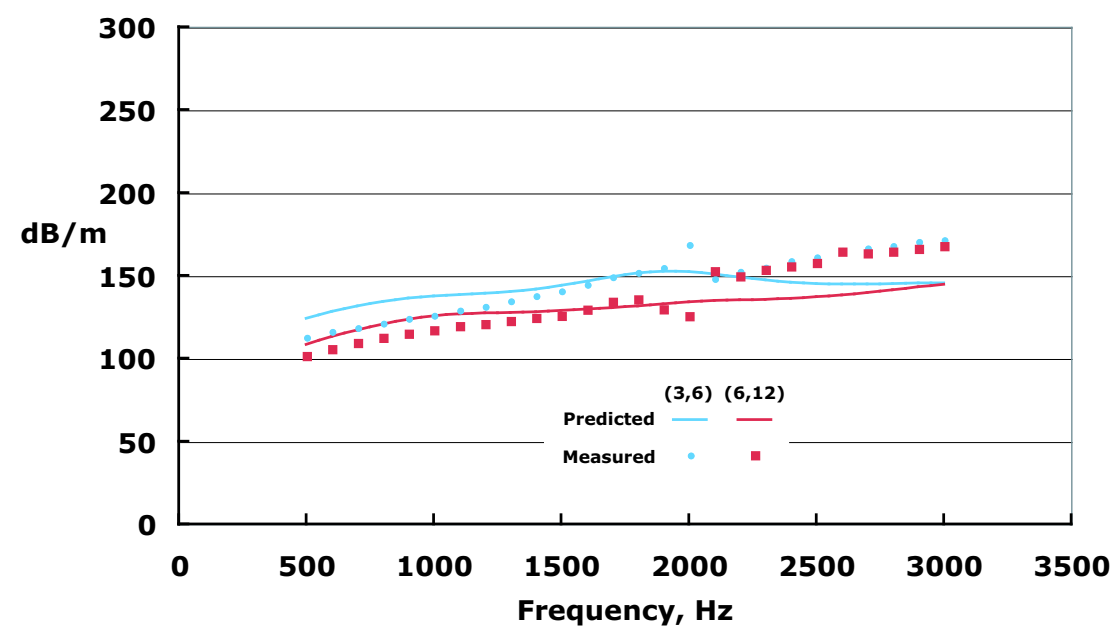

Figure 17. Attenuation rate for $(3,6)$ and $(6,12)$-layer pairs of $5 \%$ perforates, SPL of $120 \mathrm{~dB}$.

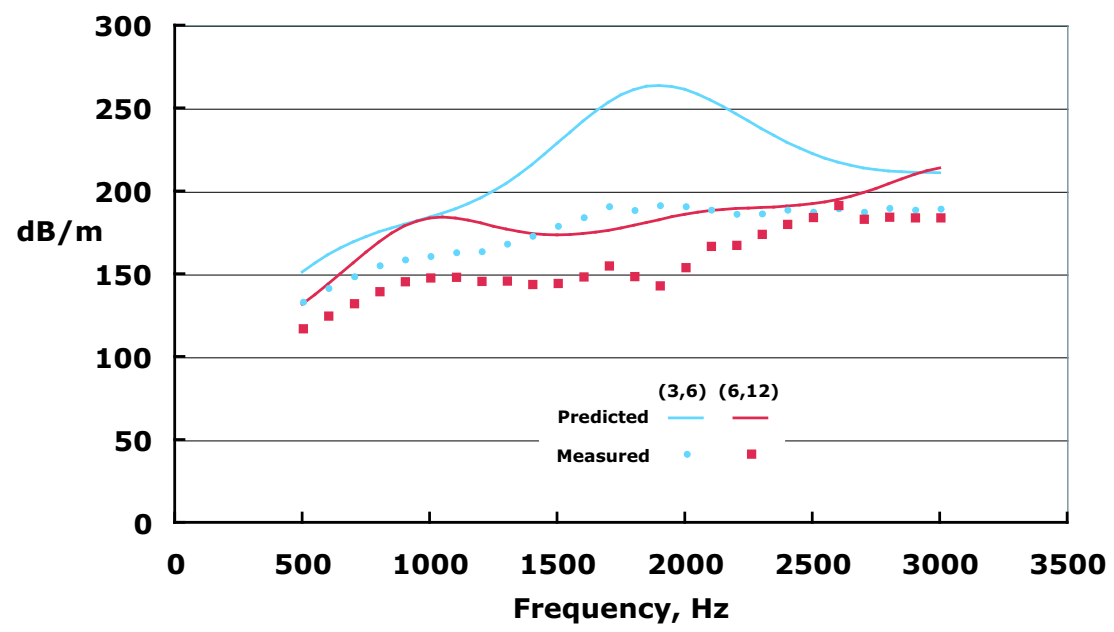

Figure 18. Attenuation rate for $(3,6)$ and $(6,12)$-layer pairs of $5 \%$ perforates, SPL of $140 \mathrm{~dB}$.

surprisingly, the predicted and measured resistance spectra trends were in excellent agreement for the three cascades. No divergent trends between predicted and measured resistance spectra were observed, as was the case for the single perforates over a $49.5 \mathrm{~mm}$ depth cavity. This suggests that the anti-resonance anomaly does not arise for the cascaded layers even though multiple "subdued" anti-resonances were clearly evident for the 6 and 12-layer cascades. Predicted resistance values were consistently less than measured values by about 0.2 to $1.0 \rho c$. The higher discrepancies were associated with the residual anti-resonance peaks and with frequency shifts of predicted and measured anti-resonance peaks that exacerbated these discrepancies. One reason for the generally improved comparisons between predicted and measured resistance spectra is that the measured resistance spectra were generally above $0.75 \rho c$ (majority well above $1.0 \rho c$ ). A second reason is that multiple perforates tend to subdue anti-resonance resistance peaks. For example, the largest anti-resonance resistance peak occurs for the 6-layer cascade at about $2100 \mathrm{~Hz}$. The predicted resistance for this peak is about $3.5 \rho c$ and the measured value is $4.4 \rho c$.

The predicted and measured reactance spectra for the three cascades were very good agreement. Data trends clustered very close to the predicted trends, with the data trend zero-crossing about 50 to $100 \mathrm{~Hz}$ lower than the predicted zero-crossings. However, these small discrepancies can be viewed as responsible for the relative frequency shifts in the residual anti-resonance peaks alluded to in the previous paragraph. 


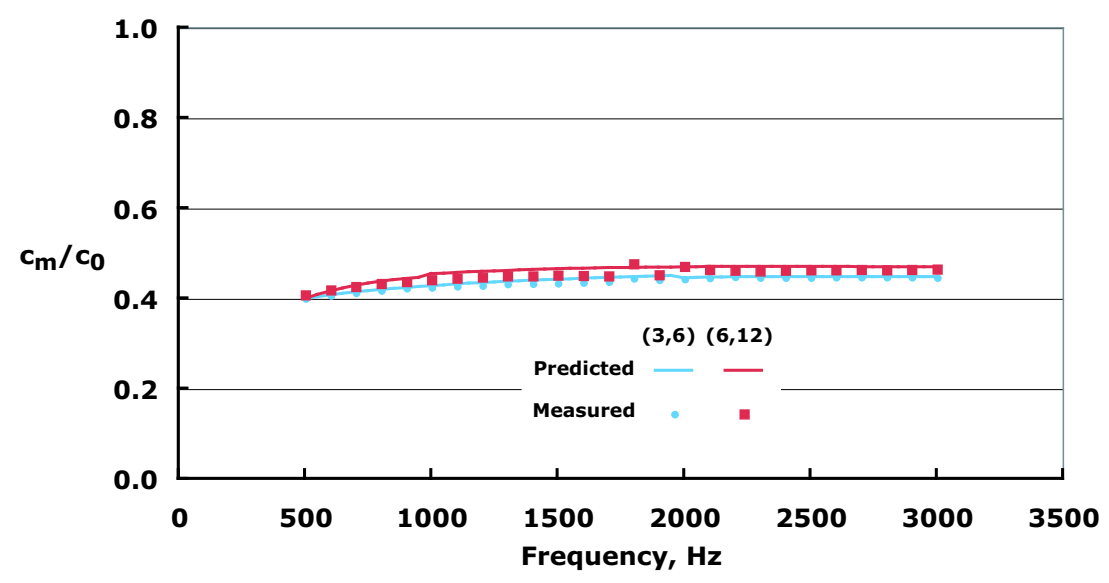

Figure 19. Sound speed ratio for $(3,6)$ and $(6,12)$-layer pairs of $5 \%$ perforates, SPL of $120 \mathrm{~dB}$.

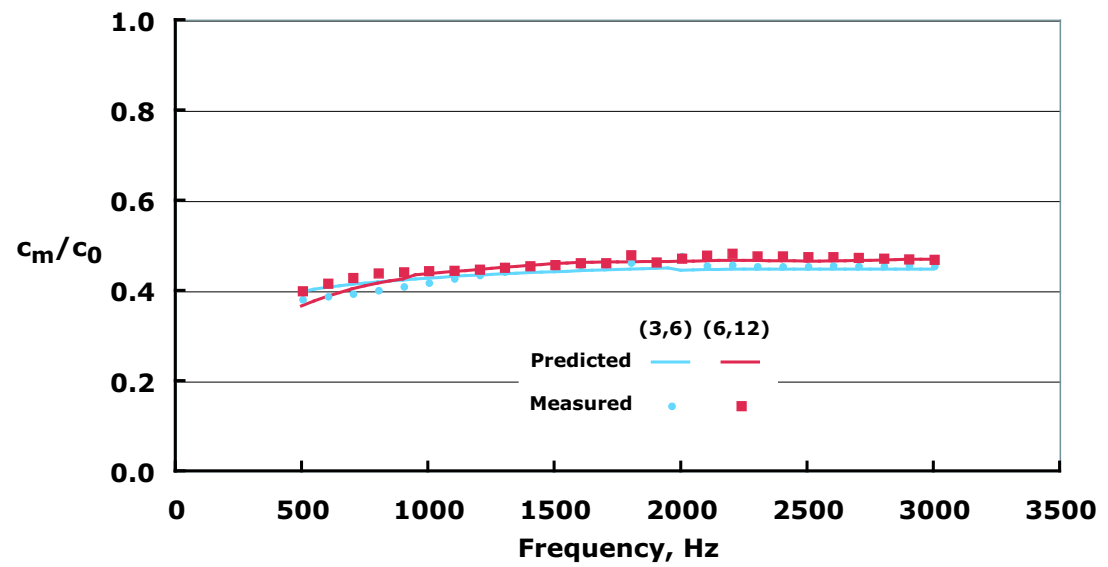

Figure 20. Sound speed ratio for $(3,6)$ and $(6,12)$-layer pairs of $5 \%$ perforates, SPL of $140 \mathrm{~dB}$.

\section{Results}

$\mathrm{C}^{\mathrm{ROM}}$ the normal incidence impedance spectra for 3,6 and 12 layer cascades, as described in the previous section, The "bulk" properties of the cascade were educed using the same procedure as discussed in the companion paper. The results are presented in the following 6 figures for the 5\% plate and for incident SPL's of 120 and $140 \mathrm{~dB}$. The graphics always present results for both the $(3,6)$-layer and $(6,12)$-layer cascades as blue and red curves/symbols, respectively. There are three graphics associated with each SPL, and these are paired in the following sequence: characteristic impedance modulus spectra for 120 and $140 \mathrm{~dB}$, respectively, attenuation in dB/m for 120 and $140 \mathrm{~dB}$, respectively, and sound speed spectra referenced to that for air for 120 and $140 \mathrm{~dB}$, respectively.

In view of the optimized TPIP performance for the 5\% plate described in a previous section, the agreement between model and data are encouraging. Model characteristic impedance modulus spectra (figures 15 and 16) generally underpredicted the measured spectra by a greater amount at $120 \mathrm{~dB}$ than at $140 \mathrm{~dB}$. The discrepancy, however, was never greater than about $0.5 \rho c$. For $120 \mathrm{~dB}$, the predicted attenuation constant spectra (figures 17 and 18) were in very good agreement with the measured spectra up to about $2000 \mathrm{~Hz}$, where a divergent trend between model and measurement is established, particularly for the $(3,6)$-layer cascade. The $(6,12)$-layer cascade prediction continues to track the data trend above $2000 \mathrm{~Hz}$, but at approximately a constant discrepancy with respect to the data trend. The data trend for the $(6,12)$-layer cascade suggests an experimental anomaly in the vicinity of $2000 \mathrm{~Hz}$, more evident at $140 \mathrm{~dB}$ than at $120 \mathrm{~dB}$. For $140 \mathrm{~dB}$ (figure 18), the story is dramatically different. For the $(3,6)$-layer cascade, the model greatly overpredicts in the mid-frequency range. However, this behavior is notably absent for the $(6,12)$-layer cascade. Excluding 
this "bump," the model over-predicts by about by about 10 to $25 \mathrm{~dB} / \mathrm{m}$ for the remainder of the frequencies. The last two figures (19 and 20) show the sound speed ratio spectra. For this parameter, the model prediction and data are in excellent agreement to within the graphical resolution.

\section{Concluding Remarks}

It has been demonstrated that the bulk parameters for a cascade of uniformly spaced perforates can be determined from an impedance prediction model for a single perforate. Generally, the results lack accuracy, but predicted trends of the bulk parameters are in reasonable agreement with experimental results. An exception to this was evident for the attenuation part of the propagation constant. For the (3,6)-layer combination at an incident SPL of $140 \mathrm{~dB}$, an attenuation rate peak of better than a $50 \mathrm{~dB} / \mathrm{m}$ increase relative to the data trend was observed. For the $(6,12)$-layer combination, this increase was not so apparent. The phase speed part of the propagation constant was in excellent agreement with experiment for both layer combinations and for all incident SPL's. In retrospect, this should not be so surprising because the impedance model prediction of perforate mass reactance was very good.

Characteristic impedance moduli tracked experimental trends very well, but generally under-predicted by about $20 \%$, or $0.5 \rho c$, out of an average of about $2.5 \rho c$. It should be noted, however, that there was significant variance between experimental results for the $(3,6)$ and $(6,12)$-layer combinations. The excellent agreement between predicted and experimental phase speed was achieved by including the perforate thickness as part of the cavity depth; i.e., the perforate thickness was assumed zero for wave propagation through the cascade. This amounted to a $22 \%$ increase in the effective air space length. This was an important correction for consistent usage of the TPIP in the cascade propagation model.

The discrepancies noted between model prediction and experiment notwithstanding, usage of the optimized TPIP model to predict the bulk parameters of a perforate cascade is encouraging. However, further work needs to be done to improve accuracy. Without these accuracy improvements, this analysis cannot be employed with confidence to discriminate between the merits of a "best" locally-reacting liner and the "hybrid" liner concept discussed in the Introduction. To that end, several recommendations are in order:

I. A high quality impedance database is needed to achieve the best possible accuracy for the perforate impedance as an isolated element. This database (for single perforate-over-cavity system) should eliminate the inaccuracy arising from the anti-resonance issue. This "contaminating" effect is a serious obstacle to developing a dynamic optimization procedure. To this end, all impedance data should be taken with the cavity presenting zero reactance by use of a variable cavity depth. Key elements for this impedance database include:

1. The excitation SPL should be extended to $160 \mathrm{~dB}$, such that nonlinear effects can be more fully evaluated.

2. The frequency range should be extended to $6000 \mathrm{~Hz}$ by usage of a smaller diameter plane-wave impedance tube (e.g., $25 \mathrm{~mm}$ diameter), such that the effects of wavelength-to-cavity depth ratio can be more fully evaluated.

3. The range of $t / d$ values should be extended. A recommended $t / d$ sequence would be $4,2,1,0.5$ and 0.25 . To better understand scale effects, these $t / d$-ratios should be achieved using different absolute values of hole diameter and length for each $t / d$ value.

4. Multiple fabrication processes (e.g., laser drilling, electron-beam drilling, punched holes) should be included, to determine the resultant effect on the model.

II. Given the high quality impedance database described above, dynamic optimization of the TPIP model should be pursued to achieve the required prediction accuracy to define the relative merits of traditional honeycomb core liners and their porous wall counterparts. Key elements of this continued development include:

1. This dynamic optimization procedure should include RMS hole-Reynolds number dependency for both the hole discharge coefficient and laminar flow constant.

2. This model should be further validated against combined broadband and tonal excitations.

\section{Acknowledgments}

The authors would like to express their appreciation to Carol Harrison and Brian Howerton for their support in the acquisition of the dataset used in this investigation. 


\section{References}

${ }^{1}$ Scott, R.A., "The propagation of sound between walls of porous material," Procedings of the Physical Society, Vol 58, Part 4, No. 328, 358-368, July, 1946

${ }^{2}$ Kurze, U.J. and Ver, I.L., "Sound Attenuation in Ducts Lined with Non-isotropic Material," Journal of Sound and Vibration, Vol 24(2), $177-187,1972$

${ }^{3}$ Jones, M.G. and Parrott, T.L., "Assessment of Bulk Absorber Properties for Multi-Layer Perforates in Porous Honeycomb Liners," AIAA2006-2403, presented at $12^{\text {th }}$ Aeroacoustics Conference, Cambridge, MA, May, 2006.

${ }^{4}$ Kraft, R.E., Yu, J. and Kwan, H.W., "Acoustic Treatment Design Scaling Methods: Volume 2: Advanced Treatment Impedance Models for High Frequency Ranges,” NASA/CR-1999-209120, Vol 2, April 999

${ }^{5}$ Motsinger, R.E. and Kraft, R.E., "Design and Performance of Duct Acoustic Treatment: Aeroacoustics of Flight Vehicles," Chapter 14, Vol 2: Noise Control, NASA Reference Publication 1258, August 1991

${ }^{6}$ Wilcox, D.C., "Basic Fluid Mechanics," First Edition, DCW Industries, Second Printing, 1998

${ }^{7}$ Coleman, Hugh W. and Steele, W. Glenn, "Experimentation and Uncertainty Analysis for Engineers," John Wiley \& Sons, 1989

${ }^{8}$ Parrott, T.L. and Jones, M.G., "Parallel-element liner impedances for improved absorption of broadband sound in ducts," Noise Control Engineering Journal, Volume 43, Number 6, November-December, 1995 